\title{
Neuropsychological deficit in haemophiliacs with human immunodeficiency virus
}

\author{
O Turnbull, M M Saling, K Kaplan-Solms, R Cohn, B Schoub
}

\begin{abstract}
Neuropsychological impairment is widely accepted as being common in acquired immunodeficiency syndrome (AIDS) but infrequent in asymptomatic human immunodeficiency virus (HIV)infected individuals. The neuropsychological function of a homogenous sample of HIV-infected haemophiliacs was investigated. Neuropsychological impairment, the nature of which is compatible with that described in the existing literature, was found in one of four AIDS cases. Eleven AIDS related complex (ARC) and 12 asymptomatic HIV-infected individuals were free of neuropsychological deficit.
\end{abstract}

The acquired immunodeficiency syndrome (AIDS) dementia complex has been generally regarded as being common in AIDS, but infrequent in asymptomatic human immunodeficiency virus (HIV) infected individuals. There has, however, recently been some controversy relating to the incidence of this disorder, specifically the incidence of neuropsychological deficit in asymptomatic HIVinfected individuals. In 1987 two reports were published $^{12}$ suggesting that the disorder occurred with high frequency at all stages of $\mathrm{HIV}$-infection, including a $44 \%$ incidence in asymptomatic HIV-infected individuals. ${ }^{1}$ These studies were criticised on methodological grounds. ${ }^{34}$

The criticisms were, firstly, that an unrealistically large proportion of "asymptomatic" subjects in the earlier studies ${ }^{12}$ were on the threshold of the later stages of immune compromise, ${ }^{3}$ suggesting that samples had not been selected in a representative manner. The studies had employed small clinical series, and had been criticised as being flawed with regard to generating epidemiological data. ${ }^{3}$ Studies which assess representative samples have been published, ${ }^{45}$ but such samples have proved difficult to obtain. The second criticism of the earlier studies ${ }^{12}$ was that the criteria used to define impairment were unsatisfactory, ${ }^{34}$ implying that individuals without HIV-related cerebral involvement were misclassified as impaired. We suggest that the strictly quantitative methodology of these studies $^{12}$ made them blind to such pseudoneurological cases. The alternative approach, diagnosis based on syndrome analysis and a qualitative approach to the interpretation of deficit, permits greater differentiation of poor performance resulting from organic conditions or due to other factors. ${ }^{6}$

The methodological problems outlined above were avoided in this study by the assessment of an entire HIV-infected population in which the date of infection was documented-made possible because infection involved a single instance of blood-borne transmission (in haemophiliacs) and, secondly, by the use of a syndrome based qualitative neuropsychological examination.

\section{Subjects}

The target population were all patients in the haemotology clinic of the Johannesburg Hospital who had contracted HIV-1 in 1982-83 from a single imported shipment of infected human anti-haemophiliac factor VIII, a possible 39 cases. Although this represents a small sample for an epidemiological study, we feel that the rare opportunity to assess a representative HIV-infected sample outweighs the disadvantages of sample size. At assessment all subjects had been HIV-infected for between five and six years. Subjects were excluded owing to 1) previous history of serious head injury (one AIDS case); 2) serious illness, with repeated hospital admission, during the study (two AIDS cases); 3) non-attendance at the clinic for the duration of the study (six asymptomatic cases); or 4) age under 14 years (three asymptomatic cases). Of the $27 \mathrm{HIV}$-infected subjects four were classified (according to standard criteria set by the Centers for Disease $\mathrm{Control}^{7}$ ) as having AIDS, 11 as AIDS related complex (ARC), and 12 as asymptomatic HIV-infected at the time of testing. In conjunction with this group 10 haemophiliacs, randomly selected from the remaining non-HIV-infected members of the clinic, were assessed as controls.

\section{Results}

The analysis of individual performances identified only one case of recognisable neuropsychological disorder. A brief summary of this case is presented below (scores on selected items are given in brackets).

Case 1. Male, mid-twenties, with AIDS. On formal testing he showed planning difficulties of moderate severity for complex material (Rey Complex Figure copy $=28$ ), a mild motor 
sequencing difficulty, an "embedded figures" effect $^{6}$ on the Block Design Subtest of the WAIS, and clear evidence of imperfect learning of complex material (Austin Maze Test ${ }^{6}$ ). These performances strongly suggest frontal involvement. The disturbance of recent memory was material non-specific (Rey Complex Figure recall $=12$ immediate, 12 delayed; Babcock Story $=10$ immediate, 12 delayed) and was responsive to cueing. Immediate memory, however, was intact (digit span $=6$ forward), and recall of simple material was good. This memory profile suggests a frontal amnesia (memory loss secondary to an organisational and planning deficit), which is compatible with the performance on the tests of executive function. He was medicated on AZT for the duration of the study, and his neuropsychological performance remained stable.

This was the only profile in the sample that suggested neuropsychological deficit. However, three other individuals scored poorly on a number of items. In these cases, described below, the poor performances did not conform to known neuropsychological syndromes, and could be ascribed to other causes.

Case 2. Male, late thirties, AIDS. The patient complained of "forgetfulness" of a few months duration but his concern with his problems, the rapid onset of symptoms, and his lucid description of the deficits seemed uncharacteristic of dementia. Other possible causes of his difficulties were suggested by the fact that he was often depressed, and in addition anxious that his colleagues at work would discover the nature of his illness. On assessment there was no evidence of a disorder of executive function. Although his memory initially appeared to be poor, his performances were inconsistent (Rey Complex Figure recall $=20, \quad 20$; but Taylor Figure recall $=10,11$ ) and inexplicable on purely neuropsychological grounds. Shortly after assessment he applied for, and was given, a pension by his employers. He reported soon afterwards that his memory had "recovered", and it has not deteriorated since. The disorder appears to have been of functional origin.

Case 3. Male, late thirties, asymptomatic HIV. This subject, a university graduate, performed well below the level indicated by his history (Rey Complex Figure recall $=16,13$; Babcock Story $=14,12)$. Despite these low scores there was often variation in performance on items which assess similar functions (Rey Complex Figure copy = 35; but Block Design $=8.5$ ) and the profile did not suggest a recognised syndrome. $\mathrm{He}$ also reported episodes of severe, sudden-onset, headache and nausea, which might have suggested cerebrovascular disorder. In no instance could any neurological or other abnormality be detected on full investigation and the symptoms underwent rapid, and complete, remission. When the subject was re-assessed some months later he had no difficulty with many of the items on which he previously performed so poorly (Taylor Recall $=24,26$; Babcock $=19,16$ ). The cause of the initially poor test scores and the physical symptoms is not clear but it is
Table Classification of Individual Cases

\begin{tabular}{lcccc}
\hline & \multicolumn{4}{l}{ Asymptomatic } \\
\cline { 2 - 5 } & Controls & $H I V$ & ARC & AIDS \\
\hline Impaired & 0 & 0 & 0 & 1 \\
Pseudoneurological & 0 & 2 & 0 & 1 \\
Total & 10 & 12 & 11 & 4 \\
$\%$ Impaired & $0 \%$ & $0 \%$ & $0 \%$ & $25 \%$ \\
\hline
\end{tabular}

likely that they resulted from functional disturbance rather than cerebral involvement.

Case 4. Male, mid-thirties, asymptomatic HIV. This subject performed poorly on almost every test, superficially suggesting generalised cognitive decline. In contrast to these findings, however, he conversed in an alert and appropriate manner, and neither he nor his family had any complaints of personality or cognitive change. Furthermore, his poor performances did not suggest a known neuropsychological syndrome, and he was clearly of below average intellect. An investigation into his history revealed a long-standing psychiatric disturbance, which appeared to be the cause of his poor performances.

A classification based on individual cases is presented in the table.

\section{Discussion}

The results of this study demonstrate that haemophiliacs with AIDS may suffer impairment of higher mental functions, as is the case for those whose infection has a sexual aetiology. A neuropsychological disorder was detected in one of four AIDS patients. Although epidemiological inferences may be highly inaccurate when drawn from a sample of this size, we nevertheless feel that these figures do not support the suggestion that neuropsychological deficits are found in the vast majority of AIDS patients. ${ }^{1}$

Secondly, neuropsychological changes were absent in all cases of ARC or asymptomatic $\mathrm{HIV}$-infection. It appears that this disorder is far less frequent in ARC and in asymptomatic $\mathrm{HIV}$-infected individuals than was suggested by earlier neuropsychological studies. ${ }^{12}$ This also supports the suggestion that the previously reported high incidence of impairment may have been due to methodological problems. ${ }^{34}$ It is unclear, however, to what extent each of the specific methodological features of this study, namely representative sampling and diagnostic criteria, contributed to the low reported incidence of deficit.

One final methodological point should be noted. Our study differs from all previous studies in this field in one respect, namely that an entirely haemophiliac sample was assessed. We feel it unlikely that this factor itself might influence the incidence and nature of AIDS dementia.

With regard to the qualitative features of the single case with a neuropsychological deficit, the patient (case 1) presented with several unequivocal frontal signs, including a frontal amnesia. This does not necessarily imply that cerebral AIDS presents primarily with anterior 
cortical involvement (which is also not supported by neuropathological evidence). The presentation could be explained in hierarchical, Jacksonian terms (in which the highest functional level suffers first in a diffuse disorder). Alternatively, it may be interpreted in terms of involvement of the extensive subcortical connections between the frontal lobes and other regions. This second mechanism has been invoked ${ }^{8}$ to explain the "frontal" presentation of subcortical dementia, which is the disorder under which the AIDS dementia complex has been classified in the literature. Thus the qualitative features of poor performance in this case are theoretically compatible with currently accepted views. It is also notable that the level of neuropsychological function in case 1 remained stable throughout the duration of the study. This may be related to the fact that he was successfully medicated with AZT.

Since submission of this paper, two other studies ${ }^{9}{ }^{10}$ have come to our attention which report incidences of neuropsychological deficit in asymptomatic HIV-infected individuals substantially lower than previously suggested. ${ }^{2}$ Their findings are comlower than previously suggested.
patible with those reported here.
1 Grant I, Hampton A, Hesselink JR, et al. Evidence of early CNS involvement in the acquired immunodeficiency
syndrome (AIDS) and other human immunodeficiency syndrome (AIDS) and other human immunodeficiency
virus (HIV) infections: Studies with neuropsychologic virus (HIV) infections: Studies with neuropsychologic testing and magnetic

2 Saykin AJ, Janssen RS, Sprehn GC, et al. Neuropsychological dysfunction in AIDS-related complex. Neurology 1987;37(suppl 1):374.

3 Price RW, Brew B, Sidtis J, et al. The brain in AIDS: Central nervous system infection and AIDS dementia complex. Science 1988;239:586-92.

4 Goethe KG, Mitchell JE, Marshall DW, et al. Neuropsychological and neurological function of Human Immunodeficiency Virus Seropositive asymptomatic individuals. Arch Neurol 1989;46:129-33.

5 Bruhn P, \& The Copenhagen Study Group of Neurological Complications of AIDS. AIDS and dementia: A quantitative neuropsychological study of unselected Danish titative neuropsychological study of unselect
patients. Acta Neurol Scand 1987;76:443-7.

patients. Acta Neurol Scand 1987;76:443-7.
6 Walsh KW. Understanding brain damage. Churchill Livingstone, 1985 .

7 Centers for Disease Control. Classification System for Human T-Lymphotrophic Virus Type III/ Lymphodenopathy-Associated Virus Infection. MMWR 1988;35:334-9.

8 Freedman M, Albert ML. Subcortical dementia. In: Vinken PJ, Bruyn GW, Klawens HL, Frederiks JAM, eds. Handbook of clinical neurology 1985;46:311-16.

9 McArthur JC, Cohen BA, Selnes OA, et al. Low prevalence of neurological and neuropsychological abnormalities in otherwise healthy HIV-l-infected individuals: Results from the Multicenter AIDS Cohort Study. Ann Neurol 1989;5:601-11.

10 Janssen RS, Saykin AJ, Cannon L, et al. Neurological and neuropsychological manifestations of HIV-1 infection:
net al. Neurological and Association with AIDS Related Complex but not asympAssociation with AIDS Related Complex but not asymp-
tomatic HIV-1 infection. Ann Neurol 1989;5:592-600. 